\title{
Characterization of Multiple Synaptic Boutons in Cerebral Motor Cortex in Physiological and Pathological Condition: Acrobatic Motor Training Model and Traumatic Brain Injury Model
}

\author{
Hyun-Wook Kim ${ }^{1}$, Ji eun $\mathrm{Na}^{1}$, ImJoo Rhyu ${ }^{1,2, *}$ \\ ${ }^{1}$ Department of Anatomy, Korea University College of Medicine, Seoul 02841, Korea \\ ${ }^{2}$ Division of Brain Korea 21 Plus Program for Biomedical Science, Korea University College of Medicine, Seoul 02841, Korea
}

*Correspondence to:

Rhyu IJ,

(iD) http://orcid.org/0000-0002-5558-6278

Tel: +82-2-2286-1149

Fax: +82-2-929-5696

E-mail: irhyu@korea.ac.kr

Received December 19, 2018

Revised December 23, 2018

Accepted December 23, 2018

\begin{abstract}
Multiple synaptic boutons (MSBs) have been reported to be synapse with two or more postsynaptic terminals in one presynaptic terminal. These MSBs are known to be increased by various brain stimuli. In the motor cortex, increased number of MSB was observed in both acrobat training (AC) model and traumatic brain injury (TBI) model. Interestingly one is a physiological stimuli and the other is pathological insult. The purpose of this study is to compare the connectivity of MSBs between AC model and TBI model in the cerebral motor cortex, based on the hypothesis that the connectivity of MSBs might be different according to the models. The motor cortex was dissected from perfused brain of each experimental animal, the samples were prepared for routine transmission electron microscopy. The 60 70 serial sections were mounted on the one-hole grid and MSB was analyzed. The 3-dimensional analysis revealed that $94 \%$ of MSBs found in AC model synapse two postsynaptic spines from same dendrite. But, 28\% MSBs from TBI models synapse two postsynaptic spines from different dendrite. This imply that the MSBs observed in motor cortex of AC model and TBI model might have different circuits for the processing the information.
\end{abstract}

Key Words: Multiple synaptic boutons (MSBs), Synaptic plasticity, Acrobat training (AC), Traumatic brain injury (TBI)

\section{INTRODUCTION}

Multiple synaptic boutons (MSBs) are one of the synaptic connecting formations that increase in multiple brain regions of various animal models. These MSBs are capable of organizing more than two postsynaptic spines with a single presynaptic bouton. Although few recent studies have analyzed these structures under a light microscopic resolution, most of the observations were examined using electron microscopy to include spatial information (Medvedev et al., 2012; Telgkamp et al., 2004; Wanaverbecq et al., 2008). Many studies reported that morphological synaptic plasticity includes the change of
MSBs during various conditions such as learning and memory (Federmeier et al., 2002; Lee et al., 2007) and brain injury (Jones, 1999). Moreover, Toni et al. previously suggested that morphological changes of MSBs are induced by longterm potentiation, a form of neuronal plasticity involved in learning and memory (Toni et al., 1999). These reports have proposed that MSBs formation provides a critical clue in neuronal plasticity, and morphological information can indicate neuronal characteristics in various conditions. However, increased number of MSBs and how they are involved in neural connection are poorly understood. Recently, Kim et al. analyzed multiple-synapse boutons (MSB) in the cerebellum of

(a) This is an open-access article distributed under the terms of the Creative Commons Attribution Non-Commercial License (http://creativecommons.org/licenses/by-nc/4.0) which permits unrestricted noncommercial use, distribution, and reproduction in any medium, provided the original work is properly cited.

Copyrights (c) 2018 by Korean Society of Microscopy 


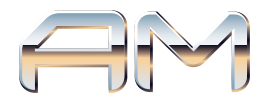

both environmentally enriched (EE) and ataxic mutant mice with 3 dimensional serial transmission electron microscopy, which revealed that MSBs from EE mice contact two spines originating from the same dendritic segments, but MSBs from tottering mice contact two spines of different dendritic origins (Kim et al., 2018).

The MSBs were found in motor cortices of acrobat trained animal and brain injury models (Greenough et al., 1985; Jones, 1999; Jones et al., 1996). We hypothesize that MSBs formation mechanism would be different according to the underlying causes as Kim et al., reported (Kim et al., 2018).

\section{MATERIALS AND METHODS}

\section{Acrobat Training Model}

Adult male Sprague-Dawley rats, 8 weeks (250 300 g), were used. They were random assigned into two groups under the following conditions: acrobat training ( $\mathrm{AC}, \mathrm{n}=8)$ model, and an inactive condition ( $\mathrm{IC}, \mathrm{n}=5$ ). All experimental procedure followed the guidelines for animal experiments edited by
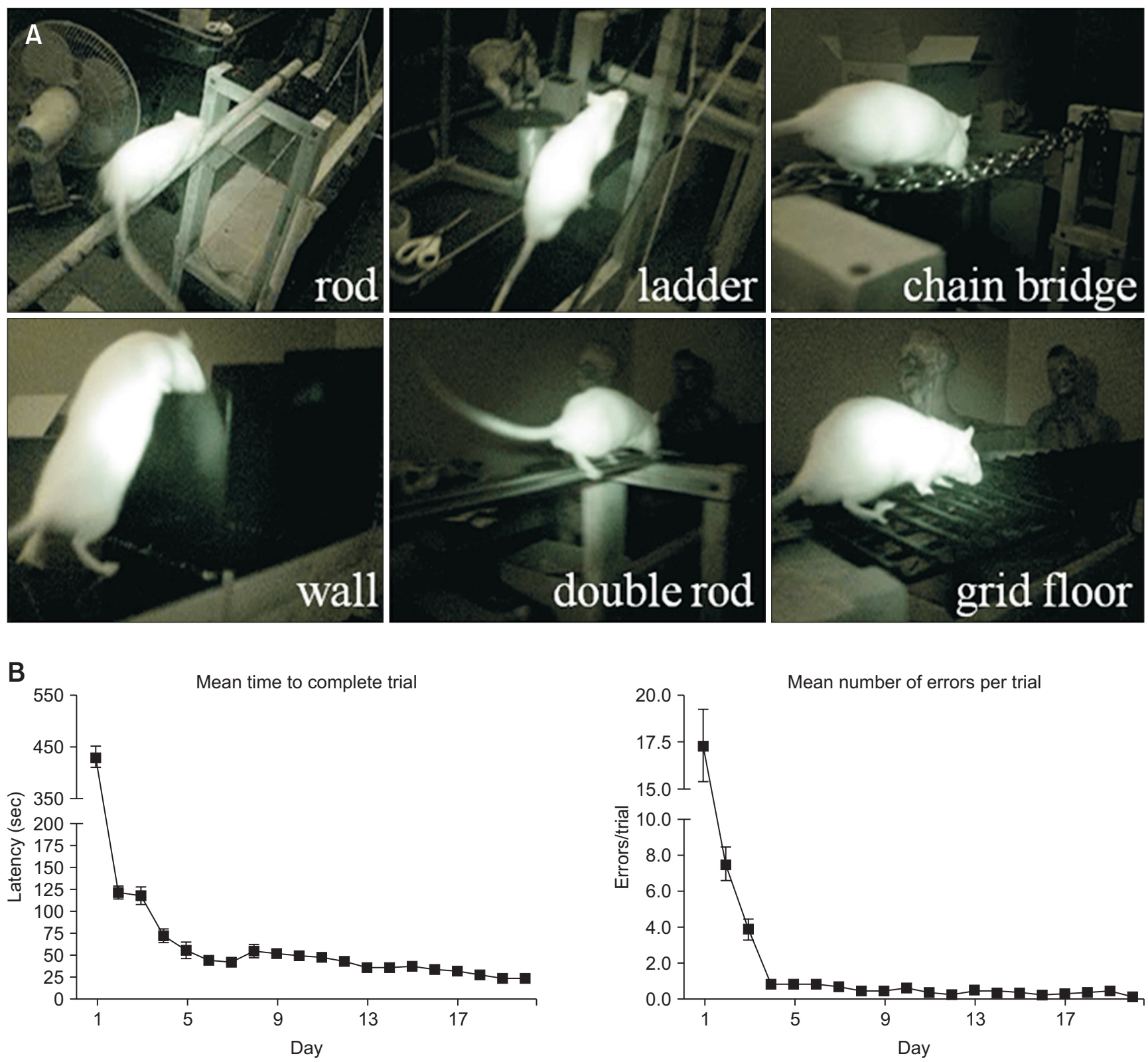

Fig. 1. Photograph of acrobat training task and mean time for AC model and error rate. (A) AC model traversing a rod, ladder, chain bridge, walls, double rod, and grid floor during 4 trials per day, for 20 days. (B) Mean time for complete a trial was significantly decreased as AC model progressed (Pearson correlation coefficient; $r=-0.95 ; \mathrm{p}<0.0001$; left). Mean number of errors per trial were also decreased during 20 days training (Pearson correlation coefficient; $\mathrm{r}=-0.5763, \mathrm{p}=0.0039$; right). 
Korea University Institutional Animal Care \& Use Committee (KUIACUC-2011-222). Animal training conditions were performed according to the procedure specified in the previous report (Lee et al., 2007). AC model were trained to run a complex course consisting of rods, ladders, chains, barriers, parallel bars, and grid platform 4 times a day for 20 days (Fig. 1A). The average time taken and the average number of errors (missed steps) per trial were recorded during each AC task (Fig. 1B). IC animals remained sedentary and received same handling for the same number of day. The correlation analysis was used to analyze the motor training performance.

\section{Traumatic Brain Injury Model}

A total number of 12 adult male C57BL/6 mice (6 weeks) were house in the standard laboratory cage and bred in a room temperature and humidity room with a 12:12 h light/ dark cycle. For cryogenic traumatic injury, adult mice were deeply anesthetized with sodium pentobarbital $(50 \mathrm{mg} / \mathrm{kg})$ and placed in a stereotaxic device (Stoelting, Wood Dale, IL), and exposed the skull carefully. After than TBI was induced by placing a steel probe $(9 \mathrm{~mm} \times 4 \mathrm{~mm})$ cooled in liquid nitrogen on the motor cortical region for 1 minute (Fig. 2), after the operation, cages with animals were placed on $37^{\circ} \mathrm{C}$ warmer for recovering from anesthesia. Animals injured by TBI were analyzed 5, 15 and 30 days after surgery.

\section{Tissue Preparation for Serial Sectioning Electron Microscopy}

Each animal was sacrificed at end of experimental duration. All experimental animals were anesthetized with sodium pentobarbital $(50 \mathrm{mg} / \mathrm{kg})$ and perfused intracardially with normal saline before TEM fixative (2\% paraformaldehyde/2.5\% glu- taraldehyde in $0.1 \mathrm{M}$ phosphate buffer; $\mathrm{pH}$ 7.4). The pre-fixed brain was removed and stored in the same fixative overnight at $4^{\circ} \mathrm{C}$.

Motor cortices (M1) were dissected from pre-fixed brain. From the olfactory bulb to $1.5 \mathrm{~mm}$ from bregma was cut in the coronal direction, and a $300 \mu \mathrm{m}$ thick section was obtained using a vibratome. The brain section was trimmed leaving only the primary motor cortex area with reference to the brain atlas and then washed with the same buffer several times. In TBI model, opposite motor cortex of the trauma insult was used for analysis.

Tissues were post-fixed with $2 \%$ osmium tetroxide for $2 \mathrm{~h}$ and en bloc stained with $0.2 \%$ uranyl acetate for $1 \mathrm{~h}$. After staining, all tissues were dehydrated through an ascending ethanol series and embedded with epoxy resin mixture. Polymerized blocks were fine trimmed into the each brain region of the blocks and 60 to 70 serial sections were cut to $70 \mathrm{~nm}$ thickness using ultramicrotome. Serial sections were laid up on carbon coated formvar grids and stained with uranyl acetate and lead citrate. Electron micrographs $(3,000 \mathrm{X} \sim 6,000 \mathrm{X})$ containing synapses randomly selected in the region of interesting were taken serially under TEM at the accelerating voltage of $80 \mathrm{kV}$ (Hitachi H-7650, Tokyo, Japan).

\section{Stereological Analysis}

For estimating the number of synapses include MSBs, we adapted previously reported stereological method and synapse number were measured per unit volume (Kim et al., 2018). Serial thin sections were collected and took picture under a TEM and the serial images were z-stack aligned using Reconstruct ${ }^{\circledR}$ software (http://synapses.clm.utexas.edu/ tools/index.stm). Three set of 20 serial sections were used for

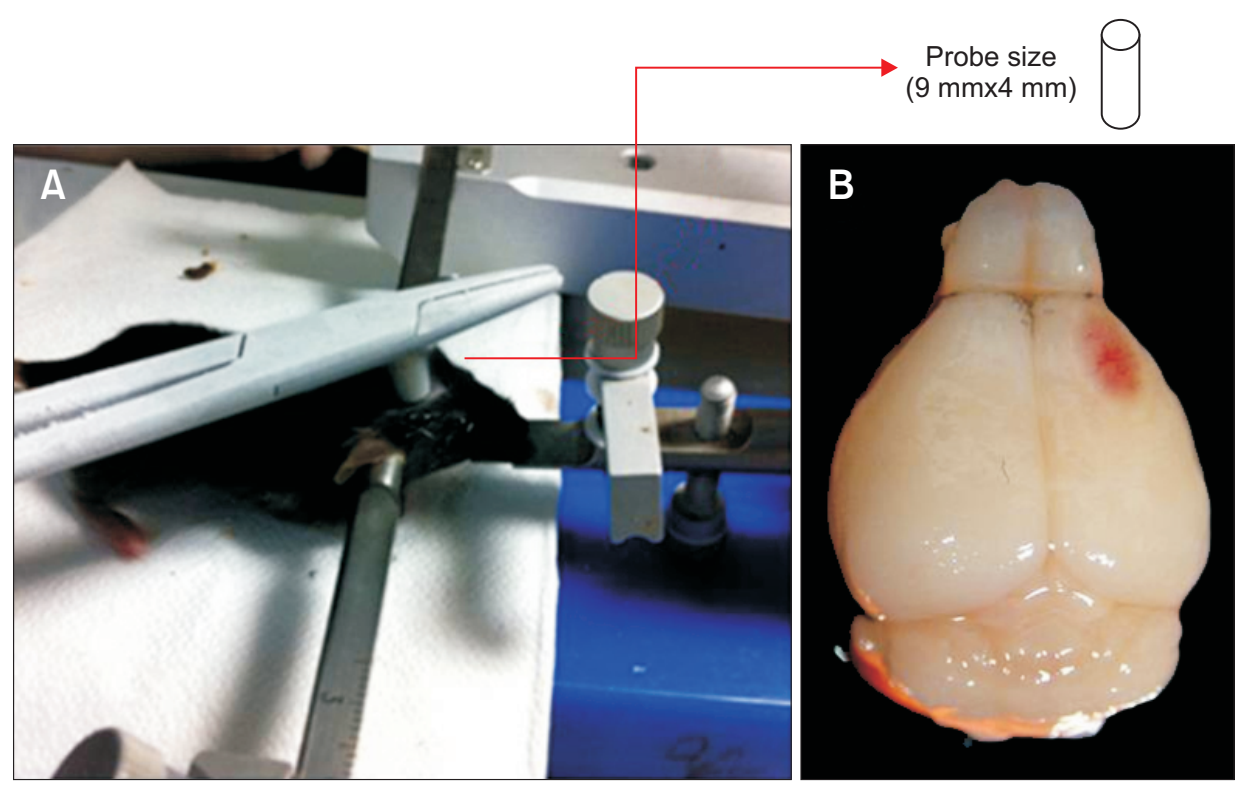

Fig. 2. Photograph of traumatic brain injury model. (A) The anesthetized mice were fixed on stereotaxic to induce TBI, and then placed in a liquid nitrogencooled probe for 1 minute in the exposed brain region. (B) The photograph of cardiac perfused mouse brain at 5 day after TBI. 
estimating synapse number using physical disector method. Counting frame size for synapse measure was $10 \mu \mathrm{m} \times 7.5 \mu \mathrm{m}$, and was randomly placed on the 'reference' section. Countable synapses were followed by the unbiased counting method (Gundersen et al., 1988). Synapses were counted on the basis of the existence of synaptic vesicle and a postsynaptic density. Synapses were counted, if a synapse on the 'reference' section was disappearing on the 'look up' section. Total number of synapses were calculated (number of synapse per unit volume; Sv) using the following formula.

$S v=$ Total number of synapses/(counting frame area $\times$ section thickness (70 nm) $\times$ number of sections)

\section{Three Dimensional Tracing of MSBs}

The MSBs in 60 70 serial thin sections per animals were took imaged serially. MSBs were identified on the presence of one presynaptic terminal such as synaptic vesicles and two or more postsynaptic spines forming postsynaptic density. 30 40 images were taken serially when defined one of single image in serial sections include identified MSBs, and most clearly image of MSBs were center section of whole serial took image. Alignment of the serial images was performed with the Reconstruct ${ }^{\mathbb{B}}$ software. Two adjacent images were overlaid in layers and bottom layer was compared and aligned with the top layer (reference section). After alignment, each post synaptic spine was traced with different colors serially when traced spine reaching to dendrite. The same dendrite MSBs (sdMSBs) spines join the same dendrite, and different dendrite MSBs (ddMSBs) spines arise from different dendrite (Fig. 3). In each animal, the number of sdMSBs and ddMSBs was presented as a percentage of total MSBs.

\section{RESULTS}

\section{Acrobat Training Task}

AC models were trained under acrobat task to identify MSBs in the cerebral motor cortex. The consistent motor training resulted in a significant decrease in the mean latency to complete a trial and number of errors per trial as training pro- gressed (Fig. 1B). These results indicate that AC groups sufficiently improved their motor activity. For statistical analysis, we used correlation analysis with days of training and time or error for each subjects. Results revealed a significant effect of day on the mean time/trial (Pearson correlation coefficient, $\mathrm{r}=-0.95, \mathrm{p}<0.0001)$ and on the mean number of errors/trial (Pearson correlation coefficient, $\mathrm{r}=-0.5763, \mathrm{p}=0.0039$ ).

\section{Motor Skill Learning Induced MSBs in Cerebral Motor Cortex}

Serial sectioning electron microscopy for stereological analysis was used to identify the distribution of MSBs in cerebral motor cortex. We examined the pattern of MSBs on the layer $\mathrm{V}$ synapse in cerebral motor cortex IC and AC. Among the total number of synapses $67.3 \pm 1.76$ in the IC, $60.73 \pm 1.57$ were SSBs and $6.6 \pm 0.4$ were MSBs (count volume, $105 \mu \mathrm{m}^{3}$; Fig. 4A and $\mathrm{B}$ ). In $\mathrm{AC}$ animals, a significant increase in the number of total synapse was observed compared to the IC animals (Total, 71.7 \pm 0.773 ; SSBs, 61.875 \pm 0.7235 ; MSBs, 9.66 \pm 0.104 ; count volume, $105 \mu^{3}$; Student t-test, $p=0.0016$; Fig. $4 \mathrm{~A}$ and B). Mean number of SSBs and MSBs were measured per unit volume $\left(\mu^{3}\right)$. Per unit volume, IC group had $0.57 \pm 0.01$ SSBs and $0.062 \pm 0.003$ MSBs. In AC animals, $0.589 \pm 0.0006$ SSBs and $0.09 \pm 0.0009 \mathrm{MSBs}$ were seen. Total number of MSBs per unit volume was increased significantly in AC animals relative to the IC group (Student t-test, $\mathrm{p}<0.0001$; Fig. $4 \mathrm{C}$ ). In the IC condition, mean proportion was $90.2 \pm 0.48 \%$ for SSBs and $9.77 \pm 0.48 \%$ for MSBs (Fig. 4D). Also, a difference in the proportion of MSBs was observed in the IC and AC conditions. Mean proportion of MSBs was $13.43 \pm 0.14 \%$ and $86.56 \pm 0.14 \%$ for the SSBs in AC condition. The outcome was in accordance with the previous reports that the motor learning increases the number of MSBs. This, perhaps, is an indication that motor skill learning induces change in the number of synapses including MSBs in motor cortex.

\section{Traumatic Brain Injury Induced MSBs in Cerebral Motor Cortex}

Then, stereological counting method with aligned serial images was used to analyze the distribution of MSBs in the cortical
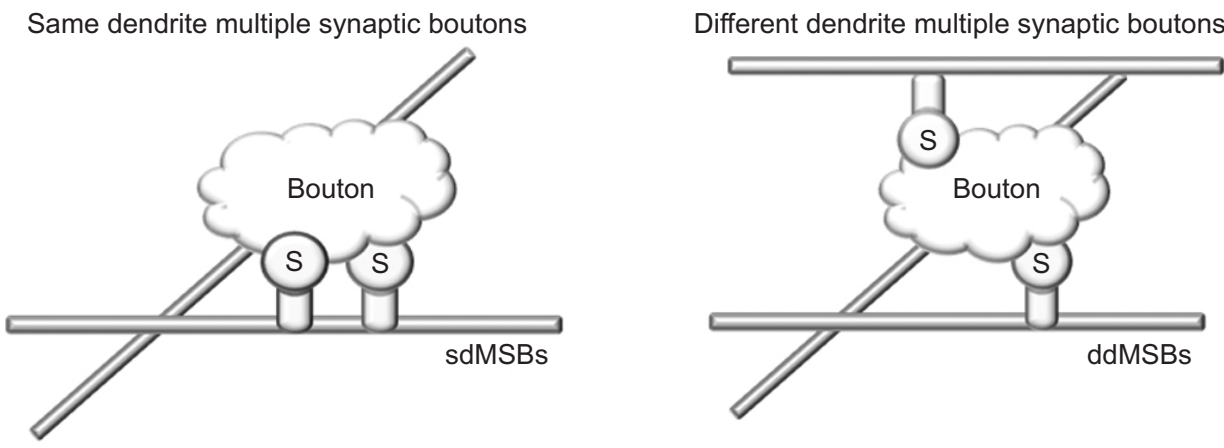

Fig. 3. Illustration for two different models of multiple synaptic boutons depend on connection. MSBs could be divided into two models, one is spine pairs arose from same dendrite (sdMSBs, left) might be enhance local synaptic efficacy, the other is spines origination from different dendrite (ddMSBs, right) suggests reorganization of neural networking. S, spine. 

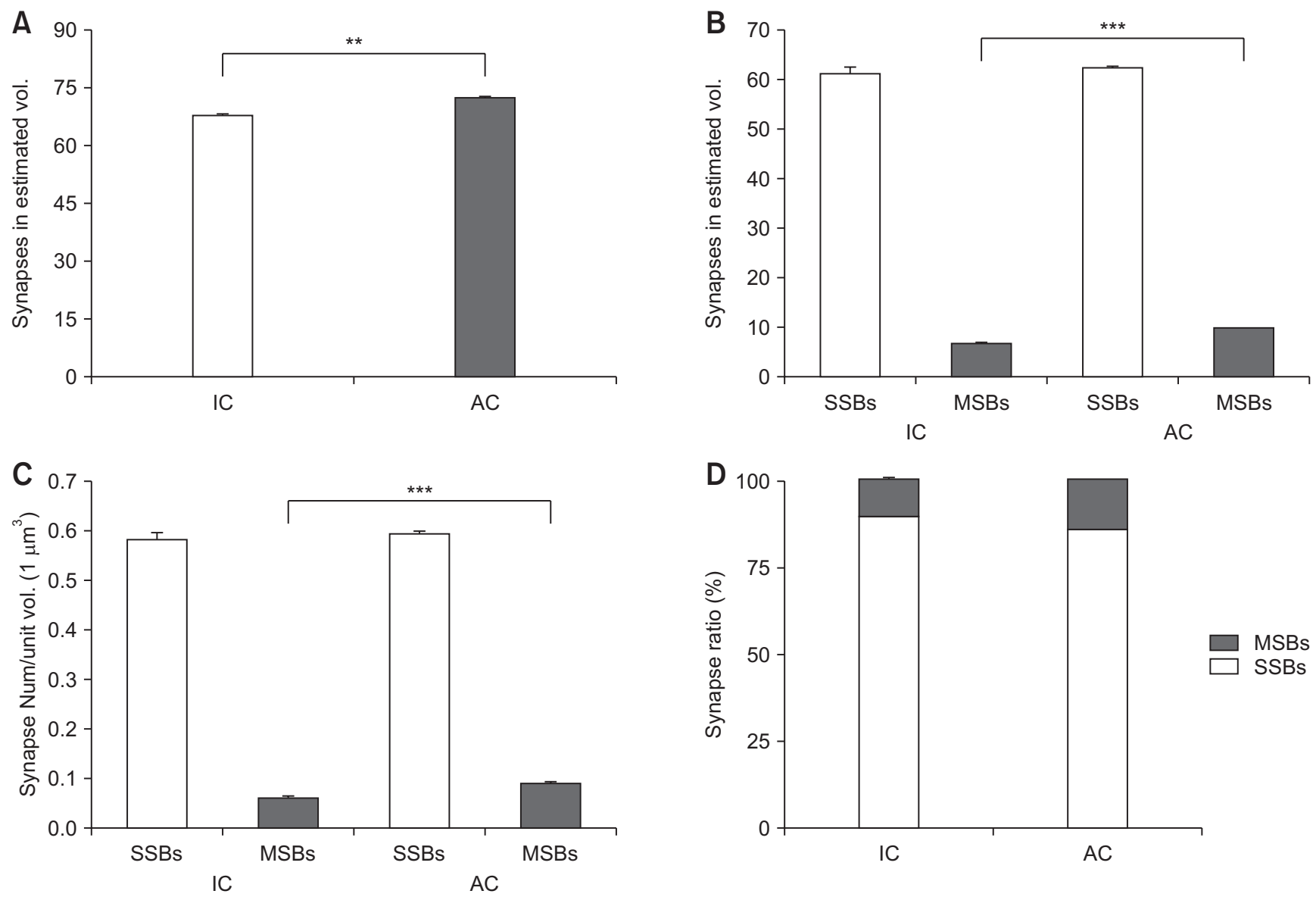

Fig. 4. Synapse distribution of cerebral motor cortex in AC model. Effects of IC and AC on the number of SSBs and MSBs. (A) Total number of synapses include SSBs with MSBs were significantly increased after AC in cerebral motor cortex layer V ( $\mathrm{p}=0.0016$; Student t-test). (B) However, effective changes number of synapses occur in MSBs (SSBs, $\mathrm{p}=0.4699$; MSBs; $\mathrm{p}<0.0001$; Student t-test). (C) Estimation for number of synapses per unit volume $\left(1 \mu \mathrm{m}^{3}\right)$ presented same results comparison total number of synapses significantly (SSBs, $p=0.4698$; MSBs, $\mathrm{p}<0.0001$; Student $\mathrm{t}$-test). (D) Proportion of SSBs and MSBs were showed similar result between IC and AC. Data are means \pm SEM.

injured mice model. For comparison with the motor training model, identical regions of the opposite site of injured cerebral cortex were used. Total number of synapses was significantly changed only at 15 day after TBI (WT, 84.22 \pm 1.46 ; TBI_5D, 89.22 $\pm 1.11, \mathrm{p}=0.0533$; TBI_15D, $91 \pm 1.52, \mathrm{p}=0.033$; TBI_30D, $86.44 \pm 0.48, \mathrm{p}=0.2244$; Student t-test; Fig. 5A). The number of MSBs increased significantly in TBI mice (WT, 8.55 \pm 0.293 ; TBI_5D, $11.2 \pm 0.22, \mathrm{p}=0.0019$; TBI_15D, $19.3 \pm 0.51, \mathrm{p}<0.0001$; TBI_30D, $13.56 \pm 0.29, \mathrm{p}=0.0003$; Student t-test; Fig. 5B). Moreover, for both groups, the number of MSBs per unit volume differed at a level of significance (WT, $0.0814 \pm 0.0028$; TBI_5D, $0.1068 \pm 0.002, \mathrm{p}<0.0001$; TBI_15D, $0.184 \pm 0.008, \mathrm{p}<0.0001$; TBI_30D, $0.129 \pm 0.003, \mathrm{p}=0.0003$; Student t-test; Fig. 5C). The TBI and WT also differed in the relative proportion of SSBs and MSBs (Fig. 5D). This implies that pathological condition of cerebral cortex induces an increase in the proportion of MSBs in opposite part of injury.

\section{Comparison of the MSBs Spine Pairs of Different Origin in Physiological - and Pathological Condition in Cerebral Motor Cortex}

Knowing that the MSBs has two or more postsynaptic spines from post neuronal dendrite, the analysis of the spine pairs originating from two different conditions in cerebral cortex was done using three dimensional tracing. $87 \pm 7.12 \%$ of MSBs observed in IC group, two spines on MSBs arose from the same dendrite in comparison with the $\mathrm{AC}$ model. The AC model showed a higher frequency of sdMSBs than ddMSBs (sdMSBs, 94 $\pm 8.42 \%$; ddMSBs, $6 \pm 1.33 \%$; Student ttest, $\mathrm{p}=0.0017$; Fig. 6). On the other hand, ddMSBs was the major component of MSBs in the TBI_15D. While the mean proportion of ddMSBs was $11 \pm 2.64 \%$ in WT mice, ddMSBs was $28 \pm 4.23 \%$ for TBI_15D mice (Student t-test, $\mathrm{p}=0.0135$; Fig. 6). These suggest that origination of spine pair in MSBs differs depending on the different neuronal connection in the physiological and pathological status in cerebral motor cortex 


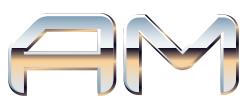

A

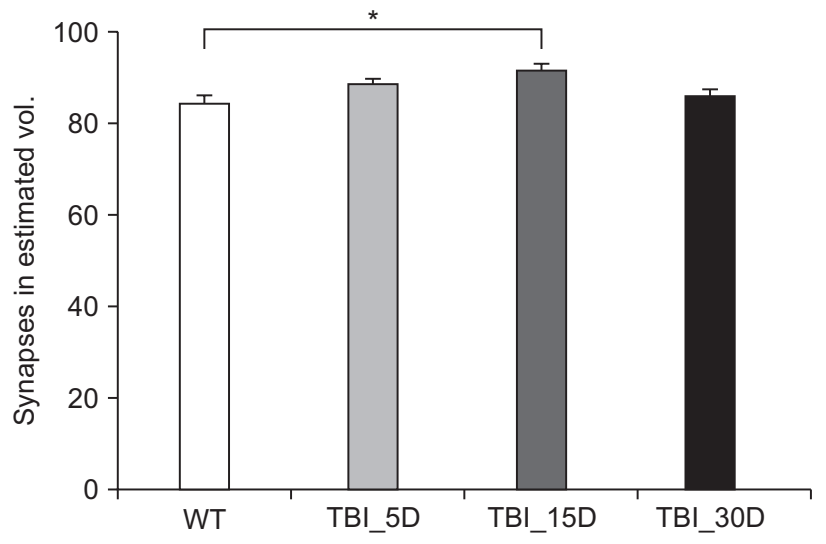

C

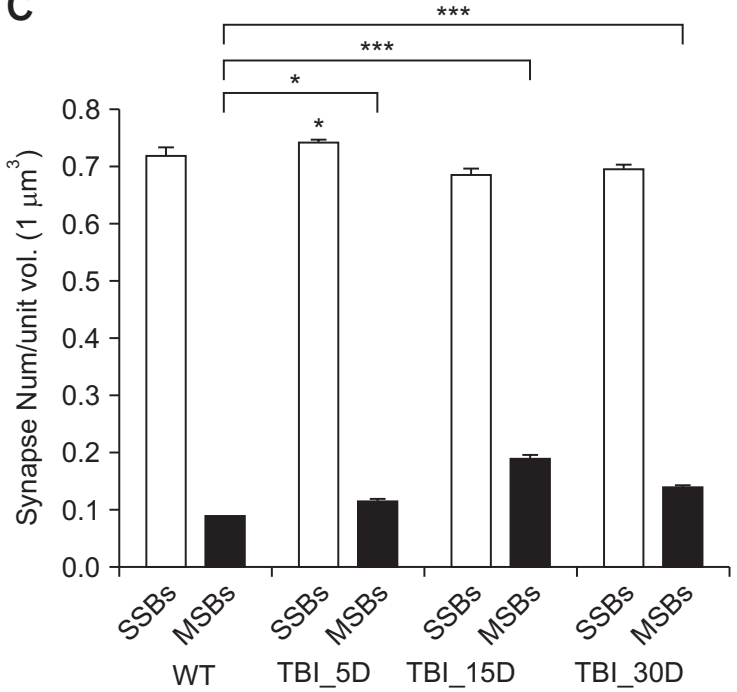

B

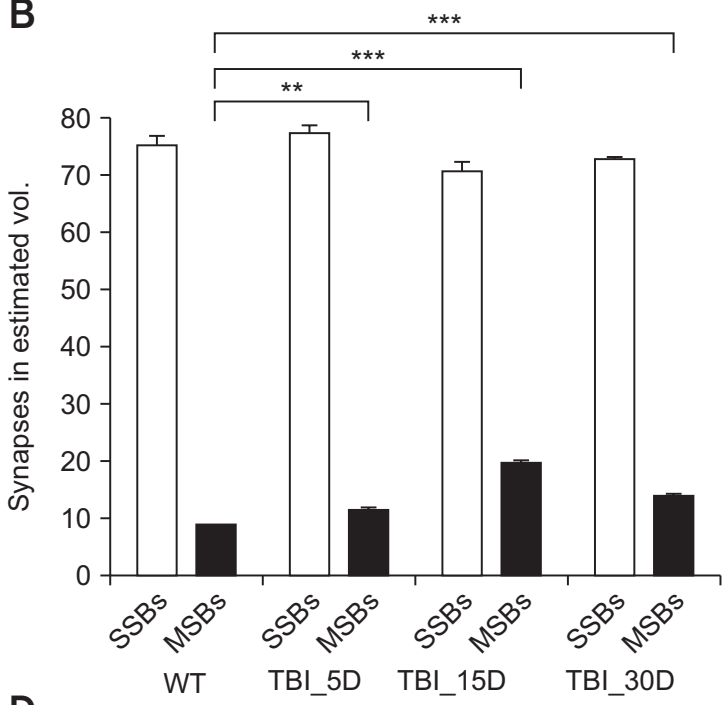

D

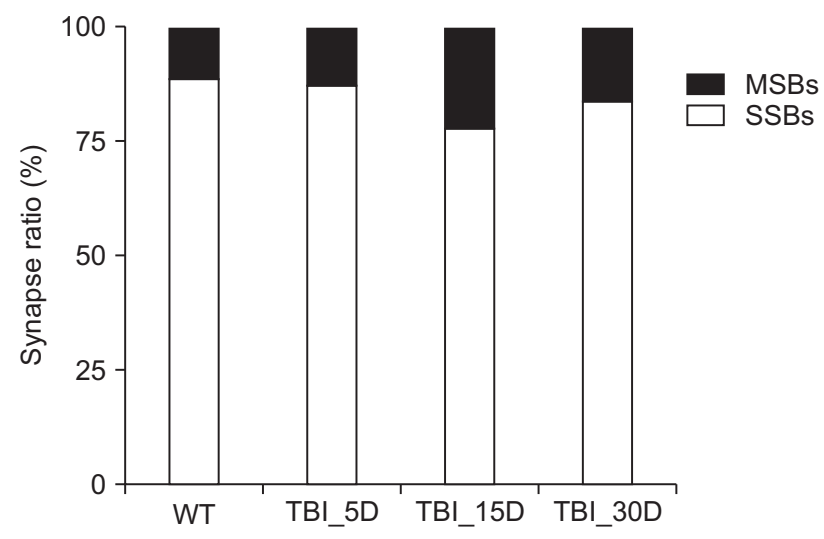

Fig. 5. Synapse distribution of cerebral motor cortex in traumatic brain injury model. Synapse distribution of WT and TBI mice in cerebral motor cortex layer V. (A) Total number of synapses includes SSBs and MSBs were changed just in after TBI 15 day model (TBI_5D, p=0.0533; TBI_15D, p=0.0330; TBI_30D, p=0.2244; Student t-test). (B) However, estimated number of MSBs were significantly increased in all TBI models (TBI_5D, p=0.0019; TBI_15D, $\mathrm{p}<0.0001$; TBI_30D, $\mathrm{p}=0.0003$; Student t-test). (C) Also, number of MSBs in per unit volume $\left(1 \mu \mathrm{m}^{3}\right)$ was significantly different in WT mice and TBI models (TBI_5D, $p=0.0019$; TBI_15D, $p<0.0001$; TBI_30D, $p=0.0003$; Student $t$-test). (D) Proportion of SSBs and MSBs were showed similar result between WT mice and TBI model analyzed per unit volume. Data are means \pm SEM.

region.

\section{DISCUSSION}

In this study we used animal models of cerebral motor cortex divided to characterization of MSBs connectivity related on neural plasticity during physiological and pathological conditions. The major finding of this study was that in each animal model, the MSB is increased in the V layer of the motor cortex, but the postsynaptic connectivity of MSB is different.

Adaptation of motor learning and external injury brought about through differential motor cortical changed by adaptations in the structure and function of the motor system. Fur- ther, the morphological change of synapse is indicated brain conditions. For example, the motor skill learning is regulated with changes in neuron morphology and synaptic strength (Greenough et al., 1985; Kleim et al., 1998; Kleim et al., 2002). Consistent with previous findings in motor learning model or motor cortical lesion model resulted in an increase in motor cortical synapses relative to normal animal (Jones, 1999; Jones et al., 1999; Kleim et al., 2002). These researches has suggested that this structural plasticity is related to functional status of motor cortex that occur synaptogenesis.

Also, increased MSBs were traced spine pair for analyze how different connectivity depend on brain condition in motor cortex layer V. In physiological condition, dramatic increased 


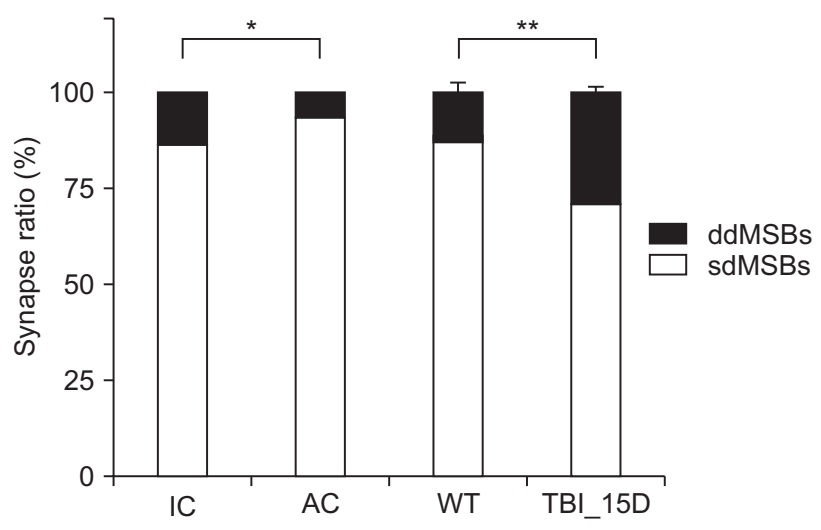

Fig. 6. Spine pairs origin of cerebral motor cortex in AC model and TBI model. The results of three dimensional tracing of MSBs for spine pair origin confirmation both two groups using Reconstruct ${ }^{\circ}$ software. Proportion of sdMSBs was significantly increased in AC model (sdMSBs, $\mathrm{p}=0.0017$; Student t-test). However, ddMSBs were major connection type of MSBs in TBI after 15 day model (ddMSBs, $\mathrm{p}=0.0135$; Student $\mathrm{t}$-test).

number of total synapses in layer $\mathrm{V}$, interestingly these increased portions were almost MSBs. These results have indicated that motor skill learning enhanced synaptogenesis process for only MSBs. In cerebellar cortex, increased number of synapse includes SSBs with MSBs (Kim et al., 2018). These new finding suggest that morphological changes of synapses may be depend on brain region. In addition, we calculated that the number of synapse change in the motor cortex layer $\mathrm{V}$ after TBI the time course in comparison with WT animal. We found that the significantly increased number of total synapses at 15 day after TBI. In contrast, MSBs were highly increased all of TBI models significantly and highest number of MSBs appeared at 15 day after TBI. Jones reported that MSBs highly existed at 30 day after lesion (Jones, 1999), which different time point came from different method for motor cortical lesion. These result suggesting that the contralateral motor cortex after ipsilateral lesion of the motor cortex enhanced new generating MSBs in layer V without SSBs.

However, we focused not only on the numerical changes of MSBs, but also how to connect multiple spines via MSBs in brain regions. Recently, Seung, H.S., proposed that analyzing the whole brain neural connection called 'connectomics' is crucial for understanding the brain function (Seung, 2011). Brain functions include motor learning and memory, or other related neuronal disorder is causes by neuronal electric signal and/or synaptic connectivity (Fujii et al., 2008). In the hippocampus, synaptic plasticity involved memorial function via neural network during active dependent function (Neves et al., 2008). These new approach may serve as an explanation to how involve to functional status by morphological change of MSBs.

We identified multiple spine origins using a serial sectioning electron microscopic approach for image tracing that provide morphological clues for synaptic plasticity and neural network. Interestingly, both animal condition was showing different synaptic connectivity via MSBs in target regions. AC model for physiological condition which increased the number of sdMSBs compared to the pathological model. These results proposed that morphological plasticity by physiological stimuli during motor skill learning may maintenance synaptic connection for existing information. In addition, these structural changes are correlation with post synaptic density size, MSBs proximity and potential compensatory weakening of synapses immediately adjacent to MSBs (Lee et al., 2013). However, TBI for pathological models showed a significant increase in the number of ddMSBs of synaptic connection in cerebral motor cortex comparison with the WT mice. Reorganization of neural network, which is one of the functional repair mechanism in the central nervous system, is required for abnormal brain condition (Dunnett, 1995).

\section{CONCLUSION}

The MSBs observed in both acrobat physiological animal model and TBI induced pathological model based were analyzed with 3-dimensional transmission electron microscopy. Major portion of connection in physiological model was MSB synapsed spines from the same dendrite, but portion of the different dendrite connection MSB was increased in pathological models. These implies that the MSBs observed in motor cortex of AC model and TBI model might have different circuits for the processing the information.

\section{CONFLICT OF INTEREST}

No potential conflict of interest relevant to this article was reported.

\section{REFERENCES}

Dunnett SB (1995) Functional repair of striatal systems by neural transplants: evidence for circuit reconstruction. Behav Brain Res. 66, 133142.
Federmeier KD, Kleim JA, Greenough WT (2002) Learning-induced multiple synapse formation in rat cerebellar cortex. Neurosci Lett. 332, 180-184. 
Fujii R, Ichikawa M, Ozaki M (2008) Imaging of molecular dynamics regulated by electrical activities in neural circuits and in synapses. Neurosignals. 16, 260-277.

Greenough WT, Larson JR, Withers GS (1985) Effects of unilateral and bilateral training in a reaching task on dendritic branching of neurons in the rat motor-sensory forelimb cortex. Behav Neural Biol. 44, 301314.

Gundersen HJ, Bagger P, Bendtsen TF, Evans SM, Korbo L, Marcussen N, Moller A, Nielsen K, Nyengaard JR, Pakkenberg B, et al. (1988) The new stereological tools: disector, fractionator, nucleator and point sampled intercepts and their use in pathological research and diagnosis. APMIS. 96, 857-881.

Jones TA (1999) Multiple synapse formation in the motor cortex opposite unilateral sensorimotor cortex lesions in adult rats. J Comp Neurol. 414, 57-66.

Jones TA, Chu CJ, Grande LA, Gregory AD (1999) Motor skills training enhances lesion-induced structural plasticity in the motor cortex of adult rats. J Neurosci. 19, 10153-10163.

Jones TA, Kleim JA, Greenough WT (1996) Synaptogenesis and dendritic growth in the cortex opposite unilateral sensorimotor cortex damage in adult rats: a quantitative electron microscopic examination. Brain Res 733, 142-148.

Kim HW, Oh S, Lee SH, Lee S, Na JE, Lee KJ, Rhyu IJ (2018) Different types of multiple-synapse boutons in the cerebellar cortex between physically enriched and ataxic mutant mice. Microscopy research and technique.

Kleim JA, Barbay S, Cooper NR, Hogg TM, Reidel CN, Remple MS, Nudo RJ (2002) Motor learning-dependent synaptogenesis is localized to functionally reorganized motor cortex. Neurobiol Learn Mem. 77, 63-77.
Kleim JA, Barbay S, Nudo RJ (1998) Functional reorganization of the rat motor cortex following motor skill learning. J Neurophysiol. 80, 33213325.

Lee KJ, Jung JG, Arii T, Imoto K, Rhyu IJ (2007) Morphological changes in dendritic spines of Purkinje cells associated with motor learning. Neurobiol Learn Mem. 88, 445-450.

Lee KJ, Park IS, Kim H, Greenough WT, Pak DT, Rhyu IJ (2013) Motor skill training induces coordinated strengthening and weakening between neighboring synapses. J Neurosci. 33, 9794-9799.

Medvedev NI, Dallerac G, Popov VI, Rodriguez Arellano JJ, Davies HA, Kraev IV, Doyere V, Stewart MG (2012) Multiple spine boutons are formed after long-lasting LTP in the awake rat. Brain Struct Funct.

Neves G, Cooke SF, Bliss TV (2008) Synaptic plasticity, memory and the hippocampus: a neural network approach to causality. Nat Rev Neurosci. 9, 65-75.

Seung HS (2011) Neuroscience: Towards functional connectomics. Nature. 471, 170-172.

Telgkamp P, Padgett DE, Ledoux VA, Woolley CS, Raman IM (2004) Maintenance of high-frequency transmission at purkinje to cerebellar nuclear synapses by spillover from boutons with multiple release sites. Neuron. 41, 113-126.

Toni N, Buchs PA, Nikonenko I, Bron CR, Muller D (1999) LTP promotes formation of multiple spine synapses between a single axon terminal and a dendrite. Nature. 402, 421-425.

Wanaverbecq N, Bodor AL, Bokor H, Slezia A, Luthi A, Acsady L (2008) Contrasting the functional properties of GABAergic axon terminals with single and multiple synapses in the thalamus. J Neurosci. 28, 11848-11861. 